\title{
Spectral Fizeau Interferometer applied to dental polymeric resins early shrinkage determination
}

\section{Interferómetro de Fizeau espectral aplicado a la determinación de la contracción temprana de resinas poliméricas dentales}

\author{
J. Antonacci1*, A. M. Mesa Yandi2,4, N. A. Russo², R. Duchowicz',4, G. F. Arenas ${ }^{1,3}$ \\ 1 Laboratorio de Laser, Instituto de Ciencia y Tecnología en Electrónica (ICyTE), Facultad de Ingeniería, \\ Universidad Nacional de Mar del Plata, Argentina. \\ 2 Centro de Investigaciones Ópticas (CONICET-CIC-UNLP), La Plata, Argentina. \\ 3 Consejo Nacional de Investigaciones Científicas y Técnicas - CONICET, Argentina. \\ 4 Facultad de Ingeniería, Universidad Nacional de La Plata, Argentina.
}

$\left(^{*}\right)$ e-mail: julian.antonacci@fi.mdp.edu.ar

Received: 20/03/2017 Accepted: 08/01/2018

DOI: $10.7149 / 0 P A .51 .1 .49076$

\begin{abstract}
:
In this work a variant of the well know Fiber Optic Fizeau Interferometer is presented. It is analyzed in the spectral domain and applied to the study of the shrinkage experienced by photocured polymeric resins. This approach, which maintains its main characteristics of being noninvasive and intrinsically self-calibrated, is sensitive to changes in the direction of evolution of the interferometric cavity length which is being measured. The Spectral Domain Fiber Optic Fizeau Interferometer generates typical response curves that must be processed in order to obtain the measurement related to the cavity length for every moment in time. The cavity is formed between the surface of the sample under test and the fiber optic tip itself. Besides, this sensor is used to determine whether or not exothermal effects from photocuring reactions affect the net shrinkage measured in the samples.
\end{abstract}

Key words: Fizeau interferometer; Dental resins; Fiber optic sensors

\section{RESUMEN:}

En este trabajo se presenta una variante del interferómetro de Fizeau en fibra óptica, analizado en el dominio espectral y aplicado al estudio de la contracción experimentada por resinas poliméricas fotocuradas. Este enfoque, que mantiene las características de ser no invasivo e intrínsecamente autocalibrado, es sensible a los cambios de sentido en la evolución de la longitud de la cavidad que se está midiendo. El Interferómetro Fizeau de Fibra Óptica en el Dominio Espectral (SDFOFI, por su sigla en inglés) genera curvas de respuesta típicas que deben ser procesadas para obtener la medida relacionada con la longitud de la cavidad en cada instante de tiempo. La cavidad está formada entre la superficie de la muestra bajo estudio y la terminación de la fibra óptica. Además, este sensor es usado para determinar si los efectos exotérmicos provenientes de la reacción de fotocurado afectan o no la contracción neta medida en las muestras.

Palabras clave: Interferómetro de Fizeau; Resinas dentales; Sensores de fibra óptica

\section{REFERENCES AND LINKS / REFERENCIAS Y ENLACES}

[1] S. Yin, P. B. Ruffin, F. T. S. Yu, Fiber Optic Sensors, Second Edition. Boca Raton, CRC Press (2008).

[2] E. Udd, W. Spillman, Fiber Optic Sensors: An Introduction for Engineers and Scientists. New Jersey, John Wiley \& Sons (2011).

[3] G. F. Arenas, S. Noriega, C. I. Vallo, R. Duchowicz, "Polymerization Shrinkage of a Dental Resin Composite Determined by a Fiber Optic Fizeau Interferometer", Opt. Comm. 271(2), 581-586 (2007).

[4] W. Schroeder, G. F. Arenas, C. I. Vallo, "Monomer conversion in a light-cured dental resin containing 1-phenyl-1,2- propanedione photosensitizer”, Polym. Int. 56(9), 1099-1105 (2007). 
[5] V. Mucci, G. F. Arenas, R. Duchowicz, W. D. Cook, C. I. Vallo, "Influence of Thermal Expansion on Shrinkage During Photopolymerization of Dental Resins Based on bis-GMA/TEGDMA", Dent. Mater. 25(1), 103-114 (2009).

[6] G. F. Arenas, S. Noriega, V. Mucci, C. I. Vallo, R. Duchowicz, "Contraction Measurements of Dental Composite Material during Photopolymerization by a Fiber Optic Interferometric Method.", AIP Conf. Proc. $992(1), 225-230$ (2008).

[7] G. F. Arenas, N. A. Russo, R. Duchowicz, Interferometric methods applied to polymeric analysis, Interferometry Principles and Applications. New York, Nova Science, 471-490, (2010).

[8] G. F. Arenas, R. Duchowicz, "Vitrification of photo-curing resins by embedded cantilever and Fizeau interferometer", Proc. of SPIE 8011, 80114Y (2011).

[9] J. Antonacci, R. Duchowicz, G. F. Arenas, "Double domain wavelength multiplexed Fizeau interferometer with high resolution dynamic sensing and absolute length detection", OLEN, 91, 227231 (2017).

\section{Introduction}

Fiber Optics Sensors are a very interesting solution in a wide range of applications in industry and laboratory areas due to its highlighted characteristics such as high sensitivity, EMI noise immunity, wideband, strength and distributed sensing capabilities. Usually, optical fiber sensors are lightweight designs, safe in explosive environments and commonly allow noninvasive measurements [1, 2]. In particular, Time Domain Fiber Optic Fizeau Interferometers (TDFOFI) were widely used by our group to study the dental polymeric shrinkage phenomenon [3]. It is well known that photo-chemical reactions within these resins are exothermic [4] and can lead to significant temperature increases [5], depending on the irradiation power of the curing light source and the reactivity from the initiator group. As a consequence, initial polymeric shrinkage dynamics may be affected if the amount of heat released from the sample which is being cured contributes with a thermal expansion level able to compete with the still early and incipient monomers shrinkage. Thus, the TDFOFI might fail to determine accurately the actual shrinkage profile, due to this expected ambiguity. The addition of embedded thermocouples into the samples could be a reasonable way to explore the thermal effects generated in the sample volume, but this classic technique does not carry conclusive results regarding some eventual thermal expansion [6], and also constitute a strange body in the volume of the sample under test. The use of embedded Bragg gratins for these cases showed coherency with general results [7] but, due to the fact that resins are normally liquid at the initial stages of the photo-polymerization process, there is no enough vitrification in the inner of the samples to adhere embedded FBGs [8]. However, it is possible to implement a Spectral Domain Fiber Optic Fizeau Interferometer (SDFOFI), using the same fiber optic elements as in the TDFOFI, but changing only the light source and the detection technique. Such device overcome any of the previously described drawbacks and measures the interferometric cavity without such ambiguity of the TDFOFI. This work shows both fiber optic Fizeau interferometer implementations, the main differences between them, their typical response signals and, as a main conclusion, the actual shrinkage curves of two very different resins. One of them is a commercial $\mathrm{P} 60-3 \mathrm{M}^{\circledR}$ restorative dental resin, and the other one is a resin in the experimentation stage developed in our laboratories.

\section{Materials \& Methods}

Figure 1 shows both implementations of the Fiber Optic Fizeau Interferometer as well as the main setup used to measure photo-polymerization shrinkage of resins.

Both sensors operate in the same way: the core of them is a fiber optic bidirectional coupler, that is a 4 arms device that splits the incident light power from the source and outputs 50/50 at the exit arms. One of them is used as the sensing probe forming an interferometric cavity between its end tip and the upper surface of the sample. The remaining exit arm is adapted in order to avoid spurious reflections. The photo-curable resin is placed in a mould made of rubber adhered to a glass plate. An intensity pattern is produced from the interference between a $4 \%$ of the light that never exits the fiber due to Fresnel reflection, and the rest that exits the fiber, reflects on the sample surface and is coupled back into the fiber. This interference signal travels along the bidirectional coupler and can be measured at the other input arm. The resins contain a photoinitiator group sensitive to the radiation in the blue region (around $470 \mathrm{~nm}$ ) and they are cured from the bottom of the array glass plate-rubber mould-resin. In all cases shown here, the power irradiation of the blue photocuring LED was set at its maximum value @ $500 \mathrm{~mA}$. 

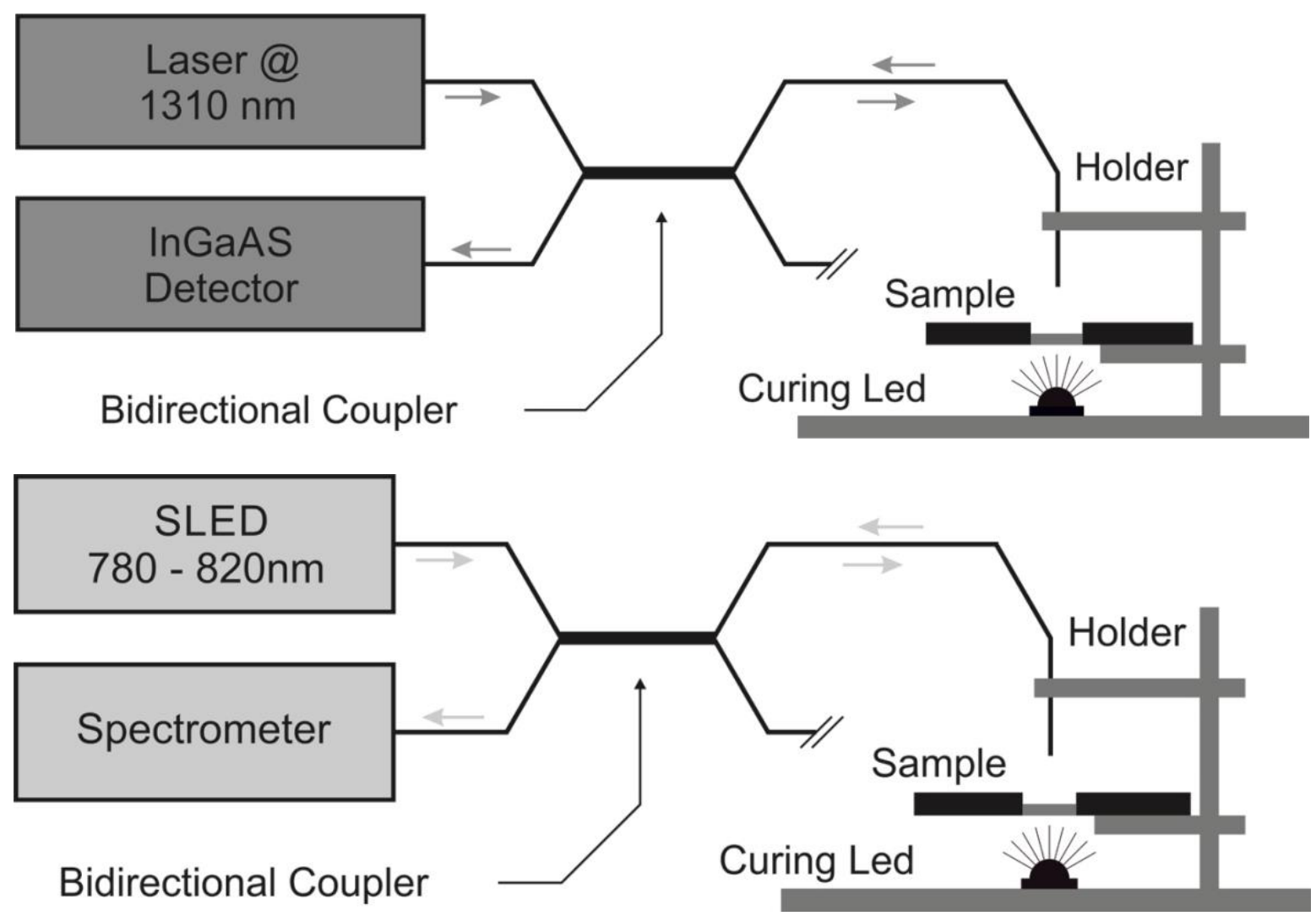

Fig. 1. Fiber Optic Fizeau Interferometer implementations used for shrinkage determination of dental resins. Up: Time Domain arrangement. Down: Spectral Domain scheme.

The TDFOFI was illuminated with a $1310 \mathrm{~nm}$ laser diode and the interference signal, typically called "interferogram", was registered by a data acquisition system with an InGaAs detector. On the other hand, for the SDFOFI we employed as broad band light source a SLED centered around $800 \mathrm{~nm}$ (nearly $40 \mathrm{~nm}$ bandwidth), and the relevant information was obtained with a CCD-based Ocean Optics HR 4000 Spectrometer. The wavelengths used for illumination in both implementations of the interferometer (1310 $\mathrm{nm}$ and $800 \mathrm{~nm})$ were chosen due to the wide availability of emitters and detectors in these spectral regions, which significantly reduces their cost. In addition, none of them alters the behavior of the material (dental resin) during the photocuring process under study, which was carried out with an irradiation at $470 \mathrm{~nm}$ as was previously mentioned.

Resins employed for the aims of this work were: the widely known $3 \mathrm{M}^{\circledR}$ restorative P60 resin, which should shrink usually a few microns and shows no thermal affects at all, and a laboratory made Bis-GMA resin mixture with 1\% camphorquinone (CQ) and Ethyl 4-(dimethylamino)benzoate (EDMAB) as the main part of the photoinitiator group. A cross section of the sample, rubber mould and glass plate is shown in Figure 2. In those cases where the resin surface did not have enough reflectivity, a tiny piece of aluminum film was deposited on top of the sample to enhance the response.

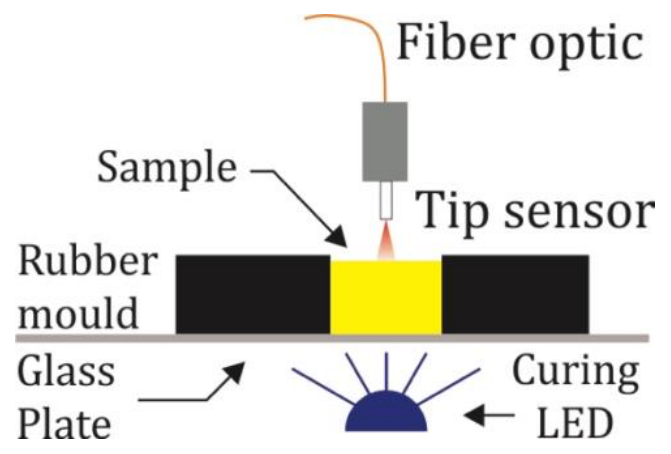

Fig. 2. Sample under study with the LED source and sensing probe. 
Figures 3 and 4 show signals obtained with both interferometers. As it can be seen in Figure 3, the TDFOFI output signal exhibits a series of maxima and minima that are related with the evolution in time of the interferometric cavity length, i.e. the sample shrinkage. As a general rule: the time between two consecutive maxima (or consecutive minima) corresponds to the time in which the cavity length increases (or decreases) a distance equal to half a wavelength of the radiation used to illuminate the interferometer $(\lambda=1310 \mathrm{~nm}$ for this case). The time scale should be adequate to the temporal evolution of the sample. Resolution of our TDFOFI is dependent on the temporal scale and the stability of the measurement set-up (in the present case it was better than $1 / 10$ of the mentioned laser wavelength).

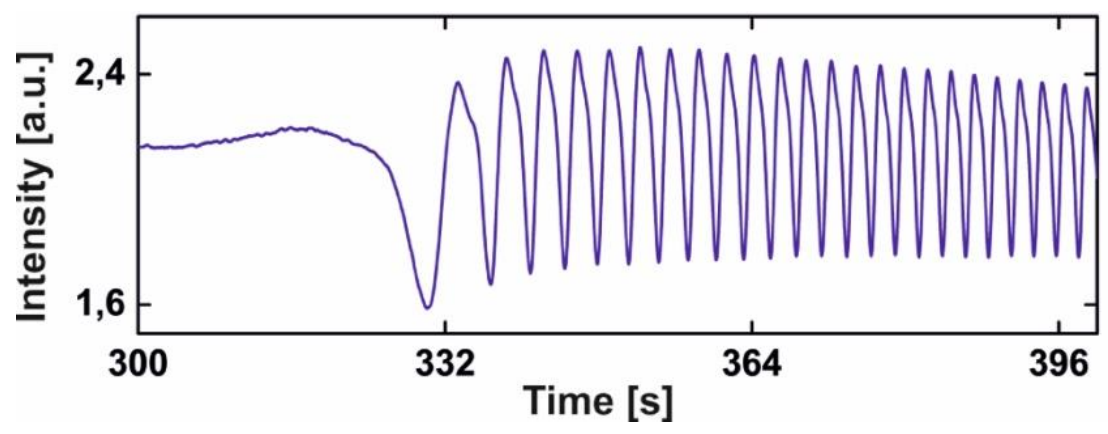

Fig. 3. Typical signal obtained in the time domain with the Fiber Optic Fizeau Interferometer during the photocuring process.

On the other hand, Fig. 4 shows three spectra from the SDFOFI implementation obtained at different times during the resin photocuring process. It can be seen that in the first seconds the change in the cavity length is very small but it increases after 50 seconds, corresponding to a contraction of the material. In this implementation of the sensor, spectra must be carefully registered as photocuring reaction proceeds. Integration time in the CCD Spectrometer must be assured in order to guarantee a proper data acquisition. In a similar way, the distance between peaks in every spectrum allows to know the evolution of the interferometric cavity length. If we call $\lambda 1$ and $\lambda 2$ the wavelengths of two consecutive peaks (corresponding to interference maxima), $f 1=\mathrm{c} / \lambda 1$ and $\mathrm{f} 2=\mathrm{c} / \lambda 2$ are the frequencies associated to each wavelength ( $c$ is the speed of the light), then the cavity length (d) is: $d=c /[2(f 1-f 2)]$ [9], allowing to obtain the cavity length from the difference between peaks. As the cavity length decreases, the interference fringes become wider, being the minimum limited by the bandwidth of the SLED because it is necessary that there be at least one period of the interferometric signal in the recorded spectrum. The resolution of the measurement is mainly dependent on the stability of spectrometer and the arithmetic used for finding the peak position.

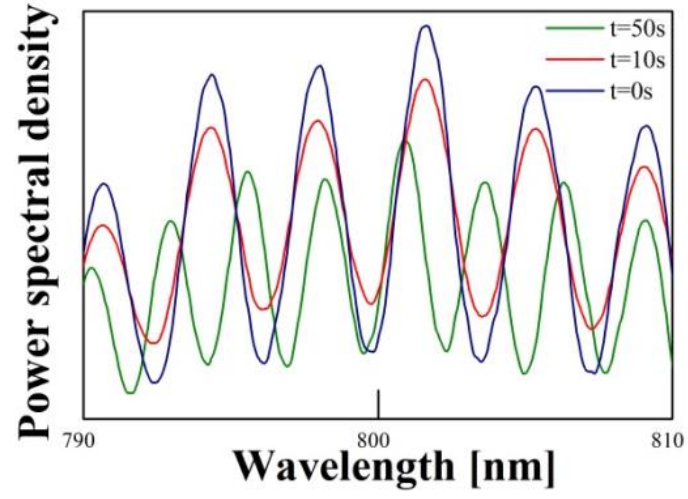

Fig 4. Three Spectral Domain FOFI plots obtained while the resin is being cured.

With SDFOFI, absolute measurements and even static measurements are possible since it is not necessary to change the cavity length in order to measure the separation between surfaces. This makes this interferometer more suitable for detecting slight thermal expansion of the sample at the very beginning of photocuring reaction. It is necessary to state here that the resolution of this setup is not so good, mainly due to the technical specifications of the spectrometer. But, for the scope of this research which involves the resins described above, the selected spectrometer worked perfectly well. 


\section{Results and discussion}

Finally, the demodulation curves obtained from the SDFOFI for different resins are shown in Figure 5. TDFOFI results are not intended to be shown here because they can be found in previous works [3,5-8], which correspond to similar resins cured by the same blue LED at similar powers.

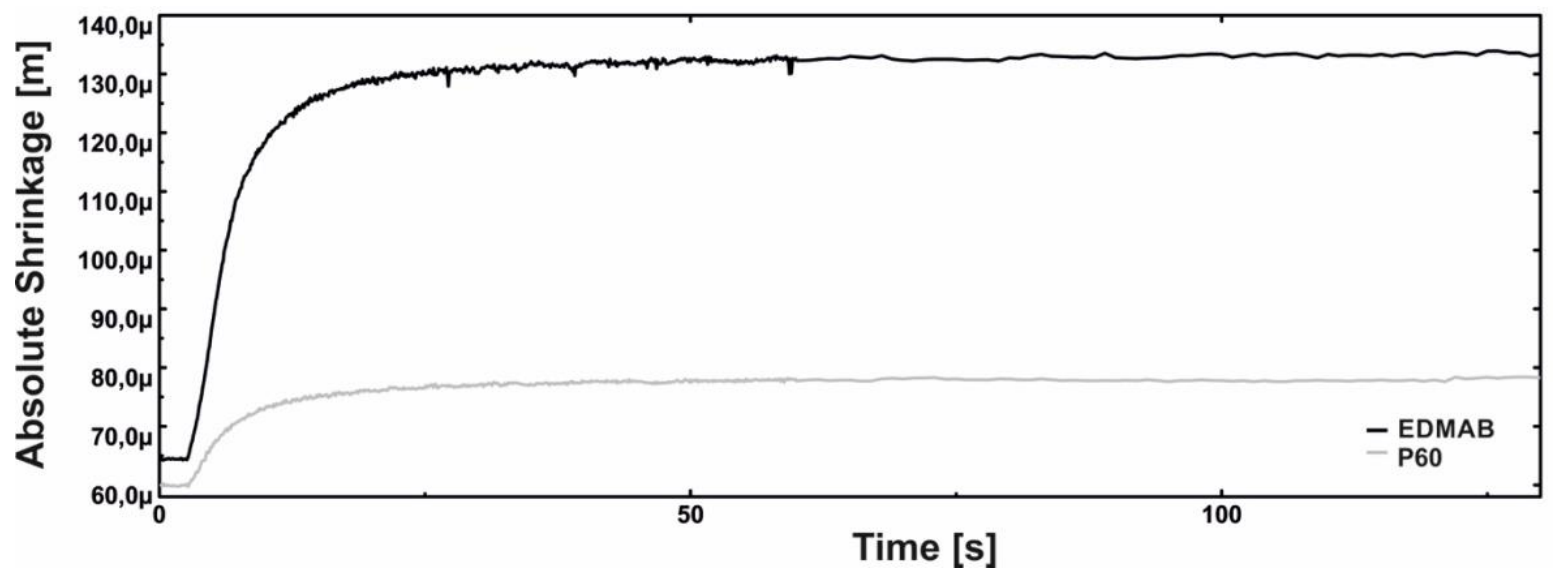

Fig. 5. SDFOFI demodulated signals for two different resins. In light gray: $3 \mathrm{M}^{\circledR} \mathrm{P} 60$ restorative dental resin; total shrinkage $=18.5$ $\mu \mathrm{m}$. In black: Bis-GMA EDMAB blend made in our laboratory; total shrinkage $=68.0 \mu \mathrm{m}$.

Comparing both curves becomes clear the performance of each formulation. The P60 resin is a commercial blend with unknown formulae, but surely it contains vitreous loads and colorants for aesthetic purposes. Thermal effects are obviously not expected to be remarkable due to biological reasons although, from previous works, we were able to measure thermal increases of few Celsius degrees above room temperature [5]. However, we decided to show these curve as a reference and control, because it is well known the behavior of such composites. Note that in nearly 20 seconds the polymerization shrinkage is almost reached and stable. Besides this normal performance, it is simple to know the initial and final distances between fiber optic tip and the resin sample upper surface. In this case, the initial cavity length was around $60 \mu \mathrm{m}$ and the net shrinkage was almost $18.5 \mu \mathrm{m}$.

Regarding the measurements made using Bis-GMA/EDMAB, significant differences can be observed. For example, it is remarkable the total shrinkage experienced by the material (about $68 \mu \mathrm{m}$ ), more than three times the one showed by the commercial P60 resin. On the other hand, it can be seen that these formulations require more time to stabilize.

It is well known that the exothermic nature of free radical bulk polymerization of dimethacrlylate monomers leads to elevated cure temperatures. Consequently, the shrinkage that occurs due to the polymerization reaction generates volumetric contraction that competes with the expansion resulting from the temperature changes. For these kinds of formulations with this sample geometry, thermal effects could be as higher as $30{ }^{\circ} \mathrm{C}$ above room temperature as it was measured in [5] using embedded thermocouples during the polymerization. With such increments in temperature, some thermal expansion might happen but, after making the measurements with the SDFOFI we can conclude that, even in those cases dealing with high reactive resins irradiated with high power curing light, thermal expansion does not take place or at least is lower than the resolution of the sensor employed and thus, would be negligible

\section{Conclusions}

Initial direction of the dimensional evolution of dental resins during photopolymerization was determined from measurements performed with the SDFOFI sensor and proper signal processing. Fizeau interferometer in its spectral version allows the researcher to know unequivocally the state and evolution of the interfering cavity at any time. In none of the cases studied here the phenomenon of thermal expansion was observed, even in cases of maximum curing irradiation power on resins with known high exothermic behavior. Although the resolution of the spectral technique is lower compared 
with the temporal version (TDFOFI), it is an interesting device that provides in a single measurement absolute position and real direction of the sample size evolution. In addition, both TDFOFI and SDFOFI could be used in conjunction to complement each other. In that way, this can yield to a better and more sophisticated technique exploiting the high spatial resolution of TDFOFI and the certainty and absolute distance determination of SDFOFI. Although the system was tested with two samples of different formulations, it can be applied to other kind of resins in the same way. It is important to mention that the illumination wavelengths used in both the temporal and the spectral implementations are only used to transmit the information, and do not generate any variation in the dimensional evolution of the sample. This is because the photoinitiator group is sensitive only to radiation in the $470 \mathrm{~nm}$ region.

\section{Acknowledgements}

This work has been supported by CONICET (PIP 112-201101-00397), Departamento de Física de la Facultad de Ingeniería - UNMdP. Facultad de Ingeniería - Universidad Nacional de la Plata (Proyecto I169) and Comisión de Investigaciones Científicas de la Provincia de Buenos Aires CIC (Resolución № 833/14), Argentina. Resins were gently prepared by researchers at INTEMA - UNMdP. 